\title{
A local smoothness criterion for solutions of the 3D Navier-Stokes equations
}

\author{
JAMEs C. Robinson $(*)$ - Witold SADOWSKi (**)
}

ABstRact - We consider the three-dimensional Navier-Stokes equations on the whole space $\mathbb{R}^{3}$ and on the three-dimensional torus $T^{3}$. We give a simple proof of the local existence of (finite energy) solutions in $L^{3}$ for initial data $u_{0} \in L^{2} \cap L^{3}$, based on energy estimates and regularisation of the initial data with the heat semigroup.

We also provide a lower bound on the existence time of a strong solution in terms of the solution $v(t)$ of the heat equation with such initial data: there is an absolute constant $\varepsilon>0$ such that solutions remain regular on $[0, T]$ if $\left\|u_{0}\right\|_{L^{3}}^{3} \int_{0}^{T} \int_{\mathbb{R}^{3}}|\nabla v(s)|^{2}|v(s)| \mathrm{d} x \mathrm{~d} t \leq \varepsilon$. This implies the $u \in C^{0}\left([0, T] ; L^{3}\right)$ regularity criterion due to von Wahl. We also derive simple a priori estimates in $L^{p}$ for $p>3$ that recover the well known lower bound $\|u(T-t)\|_{L^{p}} \geq c t^{-(p-3) / 2 p}$ on any solution that blows up in $L^{p}$ at time $T$.

The key ingredients are a calculus inequality $\|u\|_{L^{3 p}}^{p} \leq c \int|u|^{p-2}|\nabla u|^{2}$ (valid on $\mathbb{R}^{3}$ and for functions on bounded domains with zero average) and the bound on the pressure $\|p\|_{L^{r}} \leq c_{r}\|u\|_{L^{2 r}}^{2}$, valid both on the whole space and for periodic boundary conditions.

Mathematics Subject Classification (2010). 35Q30, 46E35.

KEYworDS. Navier-Stokes equations, critical spaces, calculus inequalities.

\section{Introduction}

In this paper we give an elementary proof, via simple energy-type estimates, that the three-dimensional incompressible Navier-Stokes

(*) Indirizzo dell'A.: Mathematics Institute, University of Warwick, Coventry, CV4 7AL, UK.

E-mail: J.C.Robinson@warwick.ac.uk

(**) Indirizzo dell'A.: Faculty of Mathematics, Informatics and Mechanics, University of Warsaw, Banacha 2, Poland.

E-mail: W.Sadowski@mimuw.edu.pl 
equations

$$
u_{t}-\Delta u+(u \cdot \nabla) u+\nabla p=0 \quad \operatorname{div} u=0
$$

are locally well-posed for divergence-free initial conditions $u_{0}$ that satisfy $u_{0} \in L^{2}(\Omega) \cap L^{3}(\Omega)$, where $\Omega=\mathbb{R}^{3}$ or $\Omega=[0, L]^{3}$ with periodic boundary conditions. The result goes back to Leray [15], with a more 'modern' proof presented by Fujita \& Kato [9] using the theory of semigroups (see also [3], [10], and [13]). In our paper, in which we are able to treat the case of periodic boundary conditions and the whole space in a unified way, we not only prove local existence but also estimate the local existence time from below in terms of the norm of the initial $u_{0}$ in $L^{3}$ and the properties of the solution $v(t)$ of the heat equation with the initial condition $u_{0}$ : there is an absolute constant $\varepsilon>0$ such that if

$$
\left\|u_{0}\right\|_{L^{3}}^{3} \int_{0}^{T} \int_{\mathbb{R}^{3}}|\nabla v(s)|^{2}|v(s)| \mathrm{d} x \mathrm{~d} t<\varepsilon
$$

then $u$ is smooth on $(0, T)$.

It should be noticed that for a fixed initial condition $u_{0} \in L^{3}$ we can always choose a sufficiently small time $T>0$ so that the left-hand side of (2) can be as small as we wish. Indeed, from the heat equation (4) we can easily deduce that

$$
\|v(t)\|_{L^{3}}^{3}+\int_{0}^{t} \int|\nabla v|^{2}|v| \leq\left\|u_{0}\right\|_{L^{3}}^{3}
$$

for any $t>0$. Therefore the integral on the left-hand side of (3) is finite for any $t>0$ and the assertion easily follows. We note that similar estimates where the local existence time of a strong solution for the Navier-Stokes equations depends on properties of the solution of the Stokes equations were recently proved by Farwig et al. in [8].

We will make use of the (three-dimensional) calculus inequality

$$
\|u\|_{L^{9}}^{3} \leq c \int|u \| \nabla u|^{2} \quad \text { for all } \quad u \in W^{1,3 / 2} .
$$

While this follows easily on the whole space (or in a bounded domain with zero boundary conditions) by applying the embedding $\dot{H}^{1}\left(\mathbb{R}^{3}\right) \subset L^{6}\left(\mathbb{R}^{3}\right)$ to $|u|^{3 / 2}$ (cf. Lemma 1.3 in Beirão da Veiga [1]), the proof for periodic functions with zero average requires some work (see Lemma 2 ). We note that this is a particular case of a family of inequalities: for $p<n$ and $W^{1, r}\left(\mathbb{R}^{n}\right)$, 
$r=n(p+q) /(n+q)$

$$
\|u\|_{L^{(p+q) n /(n-p)}}^{p+q} \leq c \int|u|^{q}|\nabla u|^{p} ;
$$

again, the same result holds for periodic functions with zero average.

Our analysis, which uses the method of splitting the solution into one part that satisfies the heat equation and another that deals with the nonlinear terms, is inspired by a similar approach for local existence in $H^{1 / 2}$ which can be found in the book by Chemin et al. [4], and with some simplifications in the recent paper by Marín-Rubio et al. [16]. More explicitly, we consider

$$
v_{t}-\Delta v=0 \quad v(0)=u_{0}
$$

and

$$
w_{t}-\Delta w+(u \cdot \nabla) u+\nabla p=0 \quad w(0)=0,
$$

so that $v$ is the solution of the heat equation and $u=w+v$. (One difference with the analysis in [4] is that we include all the initial data in the $v$ equation and start with zero in the equation for $w$.)

Note that one might more naturally consider instead of (4) the Stokes equation

$$
v_{t}-\Delta v+\nabla q=0, \quad \nabla \cdot v=0, \quad v(0)=u_{0} .
$$

However, on the whole space or in the case of periodic boundary conditions, which are the situations we consider here, the divergence-free property of $u_{0}$ is preserved by the heat semigroup, so the solution of (6) coincides with that of (4). In a bounded domain one would have to retain the pressure term from (6). (Another way of putting this would be to say that the Laplacian commutes with the Leray projector onto divergence-free vector fields in these cases; see Chapter Four in Constantin \& Foias [5], for example. Applying this projector to (6) one obtains (4).)

First (Theorem 1) we assume that (2) holds and that $u_{0} \in H^{2}$, so $u$ is a strong solution at least locally in time. Serrin's condition guarantees that $u$ is smooth on $(0, T)$ provided that $u \in L^{r}\left(0, T ; L^{s}(\Omega)\right)$ with

$$
\frac{2}{r}+\frac{3}{s}=1
$$

(see the nice survey paper by Galdi [10] for a modern treatment); so to prove that $u$ remains strong on the whole interval $[0, T]$, where $T$ is given by (2), we show that $u \in L^{3}\left(0, T ; L^{9}(\Omega)\right)$. 
According to Lemma 2

$$
\|u\|_{L^{9}}^{3} \leq I(u):=\int|\nabla u|^{2}|u|,
$$

and so the double integral on the left-hand side of (3) dominates the norm of $v$ in $L^{3}\left(0, T ; L^{9}\right)$ :

$$
c\|v\|_{L^{3}\left(0, T ; L^{9}\right)}^{3} \leq \int_{0}^{T} \int|\nabla v|^{2}|v| .
$$

It follows from (3) that $v \in L^{3}\left(0, T ; L^{9}\right)$. Since $u=v+w$ it remains to prove that $w \in L^{3}\left(0, T ; L^{9}\right)$.

Since

$$
\|w\|_{L^{3}\left(0, t ; L^{9}\right)}^{3} \leq c \int_{0}^{t} I(w(s)) \mathrm{d} s
$$

we only need to show that $\int_{0}^{T} I(w(s))$ ds is finite. Since $u_{0}$ is regular this integral must be finite on some interval $\left[0, T_{1}\right)$. Using relatively elementary energy estimates we derive the differential inequality

$$
\frac{\mathrm{d}}{\mathrm{d} t}\|w\|_{L^{3}}^{3}+I(w) \leq c\|w\|_{L^{3}}^{3} I(w)+c\|v\|_{L^{3}}^{3} I(v),
$$

and use a simple result for differential inequalities of the form $\dot{a}(t)+b(t) \leq c a(t) b(t)+f(t)($ Lemma 4$)$ to show that if $\int_{0}^{T}\|v\|_{L^{3}}^{3} I(v)$ is sufficiently small (which is precisely (2)) then $\int_{0}^{t} I(w)$ is uniformly bounded above by an absolute constant $1 / 2 c$ for all $t \in\left[0, T_{1}\right)$ if $T_{1} \leq T$. Thus there is no blow up for any $T_{1}<T$ and $\int_{0}^{T} I(w) \leq 1 / 2 c$.

Hence we have proved that $w$, and in consequence $u$, belongs to $L^{3}\left(0, T ; L^{9}\right)$.

In the second step of the proof (see Theorem 2) we relax our assumption about the initial condition and prove that for each divergence-free initial condition $u_{0} \in L^{3} \cap L^{2}$ satisfying (2) we can find a sequence of smooth initial conditions tending to $u_{0}$ in $L^{3}$ and giving rise to smooth solutions $u_{n}$ defined on $(0, T)$ and uniformly bounded in $L^{3}\left(0, T ; L^{9}\right)$. Then the standard procedure allows us to conclude that the limit of a subsequence of these smooth solutions is the solution of our original problem that stays bounded in $L^{3}$ and is 
smooth on $(0, T) \times \Omega$. As the sequence of initial conditions tending to $u_{0}$ we take $\mathrm{e}^{\alpha \Delta} u_{0}$, which seems natural in light of the splitting (4)-(5).

The last part of the paper is devoted to the rate of possible blow-up in $L^{p}$ spaces with $p>3$. We obtain these by deriving a lower bound on the existence time of a regular solution with $u_{0} \in L^{p}$; in contrast to condition (2) these bounds involve only absolute constants and the norm of $u_{0}$ in $L^{p}$. The calculations here are very similar to those in [1], which treats the case of the whole space; but we note that due to the generality of our Lemma 2 the proof is now valid in the periodic case also.

\section{Preliminaries}

Throughout the paper we consider the Navier-Stokes equations on a three-dimensional domain $\Omega$, where $\Omega=\mathbb{R}^{3}$ or $\Omega$ is the three-dimensional torus ${ }^{2} T^{3}=[0, L]^{3}$. When $\Omega$ is the whole space by the solution of the heat equation (4) we mean the solution given by the heat kernel:

$$
v(x, t)=\frac{1}{(4 \pi t)^{3 / 2}} \int_{\mathbb{R}^{3}} \exp \left(-\frac{|x-y|^{2}}{4 t}\right) u_{0}(y) \mathrm{d} y .
$$

We use the standard notation for the Sobolev space $H^{k}$. When $\Omega={ }^{r} T^{3}$ then by $\dot{H}^{k}$ we denote the homogeneous Sobolev space of divergence-free functions with zero mean over $\Omega$ :

$$
u=\sum_{k \in \dot{Z}_{i}^{3}} \hat{u}_{k} e^{i k x}, \quad \hat{u}_{k}=\overline{\hat{u}_{-k}}, \quad k \cdot \hat{u}_{k}=0,
$$

$\dot{Z}_{i}^{3}=Z_{i}^{3} \backslash\{0\}$, with the norm

$$
\|u\|_{\dot{H}^{s}\left(T^{3}\right)}^{2}=\sum_{k \in \dot{Z}^{3}}|k|^{2 s}\left|\hat{u}_{k}\right|^{2} .
$$

On the whole space the norm in $\dot{H}^{k}$ is given by

$$
\|u\|_{\dot{H}^{s}\left(\mathbb{R}^{3}\right)}^{2}=\int_{\mathbb{R}^{3}}|k|^{2 s}|\hat{u}(k)|^{2} \mathrm{~d} k,
$$

where

$$
\hat{u}(k)=\int_{\mathbb{R}^{3}} e^{-i k \cdot x} u(x) \mathrm{d} x .
$$


We begin with some straightforward preliminary calculations. We will use throughout the identity

$$
\partial\left(|u|^{\gamma}\right)=\gamma u_{k}\left(\partial u_{k}\right)|u|^{\gamma-2},
$$

where $\partial$ denotes any partial derivative.

We will make frequent use of the quantities $\int|u|^{\alpha-2}|\nabla u|^{2}$ and $\left.\left.\int|\nabla| u\right|^{\alpha / 2}\right|^{2}$ studied also by Beirão da Veiga in [1] (see also Galdi and Rionero [11] where a similar technique was used). The assertion of the following lemma on the whole space was already proved in [1], but for completeness we will give below the proof covering the periodic case too.

Lemma 1. For $\alpha \geq 2$, if $u \in H^{2}$ then

$$
\int-\Delta u \cdot u|u|^{\alpha-2} \geq \int|\nabla u|^{2}|u|^{\alpha-2} .
$$

Proof. Integrating by parts using (8) we obtain

$$
\begin{aligned}
& -\int\left(\partial_{i}^{2} u_{j}\right) u_{j}|u|^{\alpha-2}=\int\left(\partial_{i} u_{j}\right)\left(\partial_{i} u_{j}\right)|u|^{\alpha-2}+\int\left(\partial_{i} u_{j}\right) u_{j}(\alpha-2) u_{k}\left(\partial_{i} u_{k}\right)|u|^{\alpha-4} \\
& =\int|\nabla u|^{2}|u|^{\alpha-2}+(\alpha-2) \int\left[u_{j}\left(\partial_{i} u_{j}\right)|u|^{(\alpha / 2)-2}\right]\left[u_{k}\left(\partial_{i} u_{k}\right)|u|^{(\alpha / 2)-2}\right] \\
& \quad=\int|\nabla u|^{2}|u|^{\alpha-2}+\left.\left.\frac{4(\alpha-2)}{\alpha^{2}} \int|\nabla| u\right|^{\alpha / 2}\right|^{2}
\end{aligned}
$$

While not required for (9), note that from (8) it follows that

$$
\left.\left.|\nabla| u\right|^{\alpha / 2}\right|^{2} \leq c|u|^{\alpha-2}|\nabla u|^{2},
$$

so retaining the second term of (10) in our estimates would give us no significant extra control.

LEMma 2. Take $2 \leq p<3$. Then there exists a constant $c_{p}$ such for every $u \in W^{1, p}\left(\mathbb{R}^{3}\right)$ we have $u \in L^{3 \alpha}\left(\mathbb{R}^{3}\right)$ and

$$
\|u\|_{L^{3 \alpha}}^{\alpha} \leq c_{p} \int_{\Omega}|\nabla u|^{2}|u|^{\alpha-2},
$$

where $\alpha=p /(3-p)$. The same result is true if $\Omega$ is a bounded (perhaps periodic) domain and $u \in W^{1, p}(\Omega)$ with $\int_{\Omega} u=0$ or $\left.u\right|_{\partial \Omega}=0$. 
Note that the embedding $W^{1, p} \subset L^{3 \alpha}$ is standard. However, the norm on the right-hand side of (11) is not the $W^{1, p}$ norm. Nevertheless, it is finite for $u \in W^{1, p}$, since

$$
\int|u|^{\alpha-2}|\nabla u|^{2} \leq\left(\int|u|^{3 \alpha}\right)^{(\alpha-2) / 3 \alpha}\left(\int|\nabla u|^{3 \alpha /(1+\alpha)}\right)^{2(1+\alpha) / 3 \alpha} ;
$$

the first factor is finite since $u \in L^{3 \alpha}$ by the embedding $W^{1, p} \subset L^{3 \alpha}$, and the second factor is finite since $u \in W^{1, p}$ and $3 \alpha /(1+\alpha)=p$.

We note here that in fact a more general result holds, namely

$$
\|u\|_{L^{(p+q) /(n-p)}}^{p+q} \leq c \int|u|^{q}|\nabla u|^{p}
$$

for every $u \in W^{1, r}\left(\mathbb{R}^{n}\right)$ with $r=n(p+q) /(n+q), p<n$. Again, one can take $Q=T^{n}$ provided that $u$ has zero average.

Proof. To prove (11) on $\mathbb{R}^{3}$ assume first that $u \in C_{0}^{\infty}\left(\mathbb{R}^{3}\right)$. Then $|u|^{\alpha / 2} \in$ $H^{1}\left(\mathbb{R}^{3}\right)$, and from the Sobolev embedding $\dot{H}^{1}\left(\mathbb{R}^{3}\right) \subset L^{6}\left(\mathbb{R}^{3}\right)$ we have

$$
\|u\|_{L^{3 \alpha}}^{\alpha}=\left(\int|u|^{3 \alpha}\right)^{1 / 3}=\left\||u|^{\alpha / 2}\right\|_{L^{6}}^{2} \leq\left.\left. c \int_{\mathbb{R}^{3}}|\nabla| u\right|^{\alpha / 2}\right|^{2} .
$$

On the other hand, from (8) it follows that

$$
\left.\left.\left|\partial_{k}\right| u\right|^{\alpha / 2}\right|^{2}=\left.\left.\left|\frac{\alpha}{2} u_{i}\right| u\right|^{(\alpha-4) / 2} \partial_{k} u_{i}\right|^{2} \leq \frac{\alpha^{2}}{4}|u|^{\alpha-2}|\nabla u|^{2},
$$

so

$$
\left.\left.|\nabla| u\right|^{\alpha / 2}\right|^{2} \leq c_{\alpha}|u|^{\alpha-2}|\nabla u|^{2}
$$

and (11) follows. For $u \in W^{1, p}\left(\mathbb{R}^{3}\right)$ we use the density of $C_{0}^{\infty}\left(\mathbb{R}^{3}\right)$ in $W^{1, p}\left(\mathbb{R}^{3}\right)$.

When $\Omega$ is bounded and $\left.u\right|_{\partial \Omega}=0$ then using the Poincaré inequality the embedding $\dot{H}^{1} \subset L^{6}$ remains valid, and since $|u|^{\alpha / 2}$ is also zero on the boundary we can repeat the above argument.

However, when $\Omega$ is bounded and we only impose the zero average condition, the function $|u|^{\alpha / 2}$ does not have zero average, so we cannot apply the embedding $\dot{H}^{1}(\Omega) \subset L^{6}(\Omega)$ and we have to argue more carefully. From the embedding $H^{1}(\Omega) \subset L^{6}(\Omega)$ applied to the function $|u|^{\alpha / 2}$ we know that there is a constant $C$ such that

$$
\|u\|_{L^{3 \alpha}}^{\alpha} \leq C \int_{\Omega}|u|^{\alpha-2}|\nabla u|^{2}+C\|u\|_{L^{\alpha}}^{\alpha},
$$


where we used the fact that $\left.\left.|\nabla| u\right|^{\alpha / 2}\right|^{2}$ is bounded by $c|u|^{\alpha-2}|\nabla u|^{2}$. Now, if (11) does not hold then there exists a sequence of (non-zero) $u_{n} \in W^{1, p}$ such that

$$
\left\|u_{n}\right\|_{L^{3 \alpha}}^{\alpha} \geq n \int_{\Omega}\left|u_{n}\right|^{\alpha-2}\left|\nabla u_{n}\right|^{2}
$$

and $\int_{\Omega} u_{n}=0$.

Now we normalise the sequence $u_{n}$ in $L^{\alpha}$, setting $f_{n}=u_{n} /\left\|u_{n}\right\|_{L^{\alpha}}$. Then

$$
\left\|f_{n}\right\|_{L^{\alpha}}=1
$$

and we still have

$$
\left\|f_{n}\right\|_{L^{3 \alpha}}^{\alpha} \geq n \int_{\Omega}\left|f_{n}\right|^{\alpha-2}\left|\nabla f_{n}\right|^{2}
$$

and $\int_{Q} f_{n}=0$. Using (12) it follows that

$$
\begin{aligned}
\left\|f_{n}\right\|_{L^{3 \alpha}}^{\alpha} & \leq C \int_{\Omega}\left|f_{n}\right|^{\alpha-2}\left|\nabla f_{n}\right|^{2}+C\left\|f_{n}\right\|_{L^{\alpha}}^{\alpha} \\
& \leq \frac{C}{n}\left\|f_{n}\right\|_{L^{3 \alpha}}^{\alpha}+C,
\end{aligned}
$$

from which it follows that for all $n>C$

$$
\left\|f_{n}\right\|_{L^{3 \alpha}}^{\alpha} \leq C n /(n-C)
$$

i.e. $f_{n}$ is uniformly bounded in $L^{3 \alpha},\left\|f_{n}\right\|_{L^{3 \alpha}} \leq M$ for all $n$. It now follows from (13) that

$$
\int_{\Omega}\left|\nabla\left(f_{n}\left|f_{n}\right|^{(\alpha-2) / 2}\right)\right|^{2} \leq c \int_{\Omega}\left|f_{n}\right|^{\alpha-2}\left|\nabla f_{n}\right|^{2} \leq \frac{1}{n}\left\|f_{n}\right\|_{L^{3 \alpha}}^{\alpha} \leq \frac{M}{n} \rightarrow 0 \text { as } n \rightarrow \infty .
$$

In particular $\left\|\nabla\left(f_{n}\left|f_{n}\right|^{(\alpha-2) / 2}\right)\right\|_{L^{2}} \rightarrow 0$ as $n \rightarrow \infty$.

Notice also that $f_{n}\left|f_{n}\right|^{(\alpha-2) / 2} \in L^{2}$ with $\left\|f_{n}\left|f_{n}\right|^{(\alpha-2) / 2}\right\|_{L^{2}}=\left\|f_{n}\right\|_{L^{\alpha}}^{\alpha / 2}=1$ for every $n$. It follows that $f_{n}\left|f_{n}\right|^{(\alpha-2) / 2}$ forms a bounded sequence in $H^{1}$. One can now follows Evans (Theorem 1 in Section 5.8 in [7]) to conclude that there is a subsequence such that $f_{n_{j}}\left|f_{n_{j}}\right|^{(\alpha-2) / 2} \rightarrow F$ strongly in $L^{2}$, where $F=c$, a constant. It follows that $\|c\|_{L^{2}}=\lim _{j \rightarrow \infty}\left\|f_{n_{j}}\left|f_{n_{j}}\right|^{(\alpha-2) / 2}\right\|_{L^{2}}=1$.

Now, find a further subsequence such that $f_{n_{j}}\left|f_{n_{j}}\right|^{(\alpha-2) / 2} \rightarrow c$ a.e., so $\left|f_{n_{j}}\right|^{\alpha / 2} \rightarrow|c|$ almost everywhere. It follows that $\left|f_{n_{j}}\right|^{(\alpha-2) / 2} \rightarrow|c|^{(\alpha-2) / \alpha}$ almost everywhere, and so $f_{n_{j}} \rightarrow f:=c|c|^{(2-\alpha) / \alpha}$ almost everywhere. We also know that $\left\|f_{n_{j}}\right\|_{L^{\alpha}}=1$, and $\|f\|_{L^{\alpha}}=1$. It follows that $f_{n_{j}} \rightarrow f$ strongly in $L^{\alpha}$. 
The zero average condition is preserved by the convergence, whence $f=0$. But this contradicts the fact that $\|f\|_{L^{\alpha}}=1$, and the result follows.

The following simple result estimates the $L^{q}$ norm of the pressure in terms of the $L^{2 q}$ norm of the velocity. The result is well known in $\mathbb{R}^{3}$; we have not been able to find a standard reference in the literature for $T^{3}$, but the required periodic version of the Calderon-Zygmund Theorem can be found in the recent monograph by Shapiro [19]. It is the lack of a similar estimate in the case of a bounded domain that prevents us from proving local existence in $L^{3}$ using energy estimates for this case (cf. comments in Berselli \& Galdi [2]).

Lemma 3. Let $\Omega=\mathbb{R}^{3}$ or $\Omega=T^{3}$. If $u \in L^{2 q}(\Omega)$ is a solution of the Navier-Stokes equations then the associated pressure p belongs to $L^{q}(\Omega)$ :

$$
\|p\|_{L^{q}(\Omega)} \leq c\|u\|_{L^{2 q}(\Omega)}^{2}
$$

Proof. If we rewrite the equations in Fourier space then the $i$ th component is

$$
\frac{\mathrm{d}}{\mathrm{d} t} \hat{u}_{i}(k)+|k|^{2} \hat{u}_{i}(k)+\mathrm{i} k_{j} \widehat{u_{i} u_{j}}(k)+\mathrm{i} k_{i} \hat{p}(k)=0 .
$$

Taking the dot product with $k$ and using $k \cdot \hat{u}(k)=0(\nabla \cdot u=0)$ we obtain

$$
k_{i} k_{j} \widehat{u_{i} u_{j}}(k)=-\mathrm{i}|k|^{2} \hat{p}(k)
$$

so

$$
\hat{p}(k)=\frac{k_{i} k_{j}}{|k|^{2}} \widehat{u_{i} u_{j}}(k) .
$$

The result in $\mathbb{R}^{3}$ now follows from the Calderon-Zygmund theorem, while the result on ${ }^{\prime 3}{ }^{3}$ is a consequence of Lemma B in Chapter 6 of [19].

Finally we prove a simple ODE lemma that allows us to complete our analysis. This gives a formal version of the the 'self-consistent smallness' approach used in [4].

LEMma 4. Suppose that $x, y, \varepsilon$ are real valued, non-negative functions that are continuous on $[0, T)$ such that $x(0)=0$ and

$$
\frac{\mathrm{d} x}{\mathrm{~d} t}+y \leq c x y+\varepsilon(t) \quad \text { for all } t \in[0, T) \text { and } c>0 .
$$


If $E:=\int_{0}^{T} \varepsilon(s) \mathrm{d} s<1 / 4 c$ then

$$
\sup _{0 \leq s<T} x(s) \leq 2 E \quad \text { and } \quad \int_{0}^{T} y(s) \mathrm{d} s \leq 2 E .
$$

Proof. If $E=0$ then the assertion follows easily from Gronwall's lemma. So let us assume that $E>0$ and let

$$
Y(t)=\int_{0}^{t} y(s) \mathrm{d} s .
$$

The function $Y$ is continuous and $Y(0)=0$. Therefore to prove that $Y(t) \leq 2 E$ for all $t \in[0, T]$ it suffices to show that for all $t \in[0, T)$ we have

$$
Y(t) \leq 2 E \quad \Rightarrow \quad Y(t)<2 E .
$$

Indeed, this implication rules out the possibility that $Y(t)=2 E$ for some $t \in[0, T)$. Hence due to continuity of $Y$ no value greater than $2 E$ can be achieved.

So let us fix $t \in[0, T)$, assume that $Y(t) \leq 2 E$ and let $s \in[0, t]$. Integrating the differential inequality from 0 to $s$ yields

$$
x(s)+\int_{0}^{s} y(r) \mathrm{d} r \leq c\left\{\sup _{0 \leq r \leq s} x(r)\right\} \int_{0}^{s} y(r) \mathrm{d} r+\int_{0}^{s} \varepsilon(r) \mathrm{d} r .
$$

So for all $s \in[0, t]$ we have

$$
x(s)+Y(s) \leq c\left\{\sup _{0 \leq r \leq s} x(r)\right\} Y(s)+E .
$$

Since $x$ is continuous on $[0, t]$ there is an $s_{0} \in[0, t]$ such that

$$
\sup _{0 \leq r \leq t} x(r)=x\left(s_{0}\right) .
$$

If $Y\left(s_{0}\right)=0$ then it follows from (15) with $s=s_{0}$ that

$$
x\left(s_{0}\right) \leq E<2 E .
$$

If $Y\left(s_{0}\right)>0$ then we can use our assumption $Y\left(s_{0}\right) \leq Y(t) \leq 2 E<\frac{1}{2 c}$ to deduce from (15) that

$$
x\left(s_{0}\right) \leq 2 E-2 Y\left(s_{0}\right)<2 E .
$$


Using the assumption $Y(t) \leq 2 E<1 / 2 c$ in (15) with $s=t$ yields

$$
x(t)+Y(t) \leq \frac{1}{2} \sup _{0 \leq r \leq t} x(r)+E,
$$

so from the inequality $x\left(s_{0}\right)<E$ follows that $Y(t)<2 E$. Therefore $Y(t)<2 E$ for all $t \in[0, T)$ and the bound on $x(t)$ follows from (15).

\section{Local existence in $L^{3}$}

We will now prove smoothness of solutions of the Navier-Stokes equations on the time interval $(0, T)$, where $T$ depends only on the norm of $u_{0}$ in $L^{3}$ and on the properties of the solution of heat equation evolving from $u_{0} \in L^{2} \cap L^{3}$. In Theorem 1 we will make some additional assumptions on the regularity of $u_{0}$. These assumptions will be relaxed in Theorem 2 . We assume that $\Omega=\mathbb{R}^{3}$ or $\Omega=T^{3}$.

We note that one can find the use of similar energy-type estimates to investigate behaviour in $L^{p}$ space in the paper by Beirão da Veiga [1], and also in Berselli \& Galdi [2].

THEOREM 5. There exists an absolute constant $\varepsilon>0$ such that if $u_{0} \in H^{2}\left(\mathbb{R}^{3}\right)$ with $\nabla \cdot u_{0}=0$, and for some $T>0$

$$
\left\|u_{0}\right\|_{L^{3}}^{3} \int_{0}^{T} \int|\nabla v(t)|^{2}|v(t)| \mathrm{d} x \mathrm{~d} t<\varepsilon,
$$

where $v(t)$ is the solution of the heat equation with initial data $u_{0}$, then $u$ is smooth on $(0, T) \times \Omega$.

Proof. Since $u$ is evolving from a regular initial condition there exists a time $T^{\prime}>0$ such that $u$ is smooth on $\left(0, T^{\prime}\right)$ and $u \in L^{\infty}\left(0, T^{\prime} ; H^{2}\right)$. We want to prove that if $\varepsilon$ in (2) is sufficiently small we can choose $T^{\prime} \geq T$. Let $u=v+w$ where $v$ and $w$ are given by (4)-(5). We want to prove that $u \in L^{3}\left(0, T ; L^{9}(\Omega)\right)$. From the $v$ equation, multiplying by $v|v|$, integrating, and using Lemma 2 we obtain

$$
\frac{1}{3} \frac{\mathrm{d}}{\mathrm{d} t}\|v\|_{L^{3}}^{3}+\int|\nabla v|^{2}|v| \leq 0
$$

whence

$$
\|v(t)\|_{L^{3}}^{3}+3 \int_{0}^{t} \int|\nabla v|^{2}|v| \leq\left\|u_{0}\right\|_{L^{3}}^{3} .
$$

Therefore according to Lemma 2 we have $v \in L^{3}\left(0, T ; L^{9}(\Omega)\right)$. 
Now we want to prove that $w \in L^{3}\left(0, T ; L^{9}(\Omega)\right)$. To this end we will show that inequality (7) is satisfied on $\left[0, T^{\prime}\right)$ for all $T^{\prime} \leq T$, where $T$ is given in the statement of Theorem 1.

For the $w$ equation, multiply by $w|w|$ and integrate. Using (9)

$$
\frac{1}{3} \frac{\mathrm{d}}{\mathrm{d} t}\|w\|_{L^{3}}^{3}+\int|\nabla w|^{2}|w| \leq-\int[(u \cdot \nabla) u] \cdot w|w|-\int \nabla p \cdot w|w| .
$$

For the RHS, integrate by parts to move the derivatives off the $u$ term:

$$
\begin{aligned}
\mathrm{RHS} & =-\int u_{i} u_{j} \partial_{i}\left(w_{j}|w|\right)-\int p w_{l} \partial_{l}(|w|) \\
& =-\int u_{i} u_{j}\left(\partial_{i} w_{j}\right)|w|-\int u_{i} u_{j} w_{j} w_{k}\left(\partial_{i} w_{k}\right)|w|^{-1}-\int p w_{l} w_{k}\left(\partial_{l} w_{k}\right)|w|^{-1} \\
& \leq \int|u|^{2}\left|\nabla w \left\|\left.w\left|+\int\right| u\right|^{2}\left|w\left\|\nabla w\left|+\int\right| p\right\| w \| \nabla w\right|\right.\right. \\
& \leq \int\left|\nabla w \left\|\left.w\right|^{3}+\int|\nabla w\|w\| v|^{2}+\int|p\|w\| \nabla w|\right.\right. \\
& =: R_{1}+R_{2}+R_{3} .
\end{aligned}
$$

We estimate each of the $R_{j}$ in turn, using the notation $I(u):=\int_{\Omega}|\nabla u|^{2}|u|$.

For $R_{1}$ we use the interpolation $\|w\|_{L^{5}} \leq\|w\|_{L^{3}}^{2 / 5}\|w\|_{L^{9}}^{3 / 5}$ :

$$
\begin{aligned}
R_{1} & =\int|w|^{3}|\nabla w| \leq\left(\int|w \| \nabla w|^{2}\right)^{1 / 2}\left(\int|w|^{5}\right)^{1 / 2} \\
& \leq I(w)^{1 / 2}\|w\|_{L^{3}}\|w\|_{L^{9}}^{3 / 2} \\
& \leq c_{1} I(w)\|w\|_{L^{3}} .
\end{aligned}
$$

For $R_{2}$ we use Hölder's inequality with exponents $(2,18,9 / 4)$, and the interpolation $\|v\|_{L^{9 / 2}} \leq\|v\|_{L^{3}}^{1 / 2}\|v\|_{L^{9}}^{1 / 2}$ :

$$
\begin{aligned}
R_{2} & =\int|\nabla w\|w\| v|^{2} \\
& \leq\left(\int|w \| \nabla w|^{2}\right)^{1 / 2}\left(\int|w|^{9}\right)^{1 / 18}\left(\int|v|^{9 / 2}\right)^{4 / 9} \\
& \leq c_{2} I(w)^{2 / 3}\|v\|_{L^{9 / 2}}^{2} \\
& \leq \frac{1}{4} I(w)+c_{3}\|v\|_{L^{3}}^{3}\|v\|_{L^{9}}^{3} \\
& \leq \frac{1}{4} I(w)+c_{3}\|v\|_{L^{3}}^{3} I(v) .
\end{aligned}
$$


A local smoothness criterion for solutions of the 3D Navier-Stokes etc. 171

Finally for $R_{3}$ we use the fact that $\|p\|_{L^{9 / 4}} \leq C\|u\|_{L^{9 / 2}}^{2}$ (Lemma 3):

$$
\begin{aligned}
R_{3} & =\int|p\|w\| \nabla w| \\
& \leq\left(\int|w \| \nabla w|^{2}\right)^{1 / 2}\left(\int|w|^{9}\right)^{1 / 18}\left(\int|p|^{9 / 4}\right)^{4 / 9} \\
& \leq c_{3} I(w)^{2 / 3}\|u\|_{L^{9 / 2}}^{2} \\
& \leq \frac{1}{4} I(w)+c_{4}\|v\|_{L^{3}}^{3}\|v\|_{L^{9}}^{3}+c_{4}\|w\|_{L^{3}}^{3}\|w\|_{L^{9}}^{3} \\
& \leq \frac{1}{4} I(w)+c_{4}\|v\|_{L^{3}}^{3} I(v)+c_{4}\|w\|_{L^{3}}^{3} I(w) .
\end{aligned}
$$

The resulting inequality for $\|w\|_{L^{3}}^{3}$ is

$$
\frac{1}{3} \frac{\mathrm{d}}{\mathrm{d} t}\|w\|_{L^{3}}^{3}+\frac{1}{2} I(w) \leq c_{1} I(w)\|w\|_{L^{3}}+c_{5}\|v\|_{L^{3}}^{3} I(v)+c_{6}\|w\|_{L^{3}}^{3} I(w)
$$

Since

$$
c_{1} I(w)\|w\|_{L^{3}}=c_{1} I(w)^{2 / 3} I(w)^{1 / 3}\|w\|_{L^{3}} \leq \frac{1}{6} I(w)+c_{7} I(w)\|w\|_{L^{3}}^{3}
$$

we obtain

$$
\frac{\mathrm{d}}{\mathrm{d} t}\|w\|_{L^{3}}^{3}+I(w) \leq c_{8}\|w\|_{L^{3}}^{3} I(w)+c_{8}\|v\|_{L^{3}}^{3} I(v) .
$$

We can now apply Lemma 4 to deduce that if

$$
c_{8} \int_{0}^{T}\|v(s)\|_{L^{3}}^{3} I(v(s)) \mathrm{d} s<1 / 4 c_{8}
$$

then for any $T^{\prime} \leq T$ the quantity

$$
\sup _{0 \leq s \leq t}\|w(s)\|_{L^{3}}^{3}+\int_{0}^{t} \int_{\mathbb{R}^{3}}|\nabla w(x, t)|^{2}|w(x, t)| \mathrm{d} x
$$

is bounded on $\left[0, T^{\prime}\right)$ by $1 / 2 c_{8}$. Therefore we have proved that $w \in L^{3}\left(0, T ; L^{9}(\Omega)\right)$, and hence $u$ is smooth on $(0, T)$ where $T$ is given by (16).

We will now relax our assumption on $u_{0}$, to obtain Leray-Hopf (finite energy) type solutions for $u_{0} \in L^{2} \cap L^{3}$. For a discussion of the various 
different notions of solution if one does not assume that the energy is finite see Berselli \& Galdi [2].

Of course, on the periodic domain the requirement that $u_{0} \in L^{2}$ follows immediately from $u_{0} \in L^{3}$.

THEOREM 6. There exists an absolute constant $\varepsilon>0$ such that if $u_{0} \in L^{3} \cap L^{2}$ with $\nabla \cdot u_{0}=0$, and for some $T>0$

$$
\left\|u_{0}\right\|_{L^{3}}^{3} \int_{0}^{T} \int|\nabla v(t)|^{2}|v(t)| \mathrm{d} x \mathrm{~d} t<\varepsilon,
$$

where $v(t)$ is the solution of the heat equation with initial data $u_{0}$, then the 3D NSE has a solution $u \in L^{\infty}\left(0, T ; L^{3}\right)$. In particular:

(i) If $u_{0} \in L^{3} \cap L^{2}$ there exists a time $T>0$ such that the equations have a unique local solution $u \in L^{\infty}\left(0, T ; L^{3}\right)$; and

(ii) if $u_{0} \in L^{3} \cap L^{2}$ and $\left\|u_{0}\right\|_{L^{3}}$ is sufficiently small then the equations have a unique global solution $u \in L^{\infty}\left(0, \infty ; L^{3}\right)$.

Proof. Given $u_{0} \in L^{3} \cap L^{2}$, let $v$ be the solution of

$$
v_{t}-\Delta v=0 \quad v(0)=u_{0}
$$

and let $v_{\alpha}$ be the solution of

$$
\left(v_{\alpha}\right)_{t}-\Delta v_{\alpha}=0, \quad v_{\alpha}(0)=u_{0, \alpha}:=\mathrm{e}^{\alpha \Delta} u_{0}
$$

Note that $v_{\alpha}(t)=v(t+\alpha)$.

Since $u_{0, \alpha} \in H^{2}$ for all $\alpha>0$ we deduce from Theorem 1 that for each $\alpha$ there exists a smooth solution $u_{\alpha}$ of the Navier-Stokes equations on some time interval $\left[0, T_{\alpha}\right)$ such that

$$
u_{\alpha}(0)=u_{0, \alpha}
$$

and $T_{\alpha}$ is such that

$$
\left\|u_{\alpha}\right\|_{L^{3}}^{3} \int_{0}^{T_{\alpha}} I\left(v_{\alpha}(t)\right) \mathrm{d} t<\varepsilon .
$$

Choose any $0<T^{\prime}<T$. Let $\alpha$ be so small that $T^{\prime}+\alpha<T$. Since $v_{\alpha}(t)=v(t+\alpha)$ we have

$$
\int_{0}^{T^{\prime}} I\left(v_{\alpha}(t)\right) \mathrm{d} t=\int_{\alpha}^{T^{\prime}+\alpha} I(v(t)) \mathrm{d} t \leq \int_{0}^{T} I(v(t)) \mathrm{d} t .
$$


A local smoothness criterion for solutions of the 3D Navier-Stokes etc. 173

Since

$$
u_{0, \alpha} \rightarrow u_{0}
$$

as $\alpha \rightarrow 0$ in $L^{3}$ we can choose $\alpha_{0}$ such that for all $\alpha<\alpha_{0}$ we have

$$
\left\|u_{\alpha}\right\|_{L^{3}}^{3} \int_{0}^{T^{\prime}} I\left(v_{\alpha}(t)\right) \mathrm{d} t<\varepsilon .
$$

It follows that for every $0<T^{\prime}<T$ we can find a sequence of smooth solutions of the Navier-Stokes equations defined on time interval [0, $T^{\prime}$ ) such that $u_{\alpha}(0)=u_{0, \alpha}$ and $u_{\alpha}$ are uniformly bounded in $L^{3}\left(0, T^{\prime} ; L^{9}\right)$. From wellknown results on the uniqueness of solutions satisfying Serrin's condition in the class of all weak solutions satisfying the energy inequality it follows that $u_{\alpha}$ can be obtained by standard Galerkin approximations and that for sufficiently small $\alpha$ we have

$$
\sup _{0 \leq t \leq T^{\prime}}\left\|u_{\alpha}(t)\right\|_{L^{2}}^{2}+\int_{0}^{T^{\prime}}\|\nabla u(t)\|_{L^{2}}^{2} \mathrm{~d} t \leq 2\left\|u_{\alpha, 0}\right\|_{L^{2}}^{2} \leq 2\left\|u_{0}\right\|_{L^{2}}^{2}+1 .
$$

Hence $u_{\alpha}$ are uniformly bounded in $L^{\infty}\left(0, T^{\prime} ; L^{2}\right)$ and $\nabla u_{\alpha}$ are uniformly bounded in $L^{2}\left(0, T^{\prime} ; L^{2}\right)$ Therefore we can choose a sequence of solutions bounded in $L^{2}\left(0, T^{\prime} ; H^{1}\right)$ and strongly convergent in $L^{2}\left(0, T^{\prime} ; L^{2}(\Omega)\right.$ ) (or, in case of $\Omega=\mathbb{R}^{3}$ in $L^{2}\left(0, T^{\prime} ; L^{2}\left(\Omega^{\prime}\right)\right)$ for any bounded $\left.\Omega^{\prime} \subset \mathbb{R}^{3}\right)$. The limit function $u$ is the smooth solution of the Navier-Stokes equations defined on time interval $\left(0, T^{\prime}\right)$ and satisfying initial condition $u_{0}$. Since $T^{\prime}<T$ was arbitrary the result follows.

Global existence for small data follows similarly; from (17) we know that for any $t>0$

$$
\int_{0}^{t} I(v(s)) \mathrm{d} s \leq\left\|u_{0}\right\|_{L^{3}}^{3}
$$

whence (2) gives existence for all $t \geq 0$ provided the condition

$$
\left\|u_{0}\right\|_{L^{3}}^{6}<\varepsilon .
$$

is satisfied

Using our regularity criterion (2) we can give a simple proof of the $C^{0}\left([0, T] ; L^{3}\right)$ regularity condition of von Wahl [20] (also derived by Giga [12]). However, note that our solutions have finite energy; regularity under the assumption $u \in C^{0}\left([0, T] ; L^{3}\right)$ is shown for very weak solutions (also on bounded domains) by Berselli \& Galdi [2]. 
Corollary 7. Suppose that $u$ is a weak (Leray-Hopf) solution of the Navier-Stokes equations with $u \in C^{0}\left([0, T] ; L^{3}\right)$. Then $u$ is regular on $(0, T]$.

Proof. Let $M=\max _{t \in[0, T]}\|u(t)\|_{L^{3}}$.

Note that if $v_{t}(\cdot)$ is the solution of the heat equation with initial condition $u(t) \in L^{3}$ then

$$
\|S(\tau) u(t)\|_{L^{3}}^{3}+\int_{0}^{\tau}\left|v_{t}(s)\left\|\left.\nabla v_{t}(s)\right|^{2} \mathrm{~d} s=\right\| u(t) \|_{L^{3}}^{3} ;\right.
$$

thus

$$
\begin{aligned}
\|u(t)\|_{L^{3}}^{3} & \int_{0}^{\tau}\left|v_{t}(s) \| \nabla v_{t}(s)\right|^{2} \mathrm{~d} s \leq M^{3}\left(\|u(t)\|_{L^{3}}^{3}-\|S(\tau) u(t)\|_{L^{3}}^{3}\right) \\
& \leq M^{3}\left(\|u\|_{3}-\|S(\tau) u\|_{3}\right)\left[\|u\|_{3}^{2}+\|u\|_{3}\|S(\tau) u\|_{3}+\|S(\tau) u\|_{3}^{2}\right] \\
& \leq 3 M^{5}\left(\|u\|_{3}-\|S(\tau) u\|_{3}\right),
\end{aligned}
$$

since $\|S(t) u\|_{L^{3}} \leq\|u\|_{L^{3}}$.

We now show that there exists a $\tau>0$ such that

$$
\|u(t)-S(\tau) u(t)\|_{L^{3}}<\varepsilon:=\varepsilon_{0} / 3 M^{5} \quad \text { for every } \quad t \in[0, T] .
$$

If not then there exist $t_{n} \in[0, T]$ and $\tau_{n} \rightarrow 0$ such that

$$
\left\|u\left(t_{n}\right)-S\left(\tau_{n}\right) u\left(t_{n}\right)\right\|_{L}^{3} \geq \varepsilon .
$$

Extracting a subsequence and relabelling we can guarantee that $t_{n} \rightarrow$ $t^{*} \in[0, T]$. But, noting that

$$
\begin{aligned}
\left\|S\left(\tau_{n}\right) u\left(t_{n}\right)-u\left(t^{*}\right)\right\|_{3} & \leq\left\|S\left(\tau_{n}\right)\left[u\left(t_{n}\right)-u\left(t^{*}\right)\right]+\left[S\left(\tau_{n}\right) u\left(t^{*}\right)-u\left(t^{*}\right)\right]\right\|_{3} \\
& \leq\left\|u\left(t_{n}\right)-u\left(t^{*}\right)\right\|_{3}+\left\|S\left(\tau_{n}\right) u\left(t^{*}\right)-u\left(t^{*}\right)\right\|_{3},
\end{aligned}
$$

it follows from the assumed continuity of $u(\cdot)$ and the strong continuity of $S(\cdot)$ (see the notes by Rodriguez-Bernal [17], for example) that $S\left(\tau_{n}\right) u\left(t_{n}\right) \rightarrow u\left(t^{*}\right)$. So we can take the limit as $n \rightarrow \infty$ in (18) to deduce that $0=\left\|u\left(t^{*}\right)-u\left(t^{*}\right)\right\|_{3} \geq \varepsilon$, which is clearly absurd.

It follows that there exists a $\tau>0$ such that

$$
\|u(t)\|_{L^{3}}^{3} \int_{0}^{\tau}\left|v_{t}(s) \| \nabla v_{t}(s)\right|^{2} \mathrm{~d} s<\varepsilon_{0}
$$

for every $t \in[0, T]$, and in particular $u$ is regular on $(0, T+\tau)$. 
The proof of regularity when $u \in L^{\infty}\left(0, T ; L^{3}\right)$ due to Escauriaza et al. [6] is significantly more involved. It is perhaps tantalising to note that under this condition $u$ is right continuous into $L^{3}$ (see for example Lemma 7.4 in Galdi [10]); but this is not sufficient to obtain the required contradiction from (18).

\section{Lower bounds on solutions that blow up in $L^{p}$}

In Theorem 2 the existence time $T$ depends not only on the norm of $u_{0}$ in $L^{3}$ but also on the properties of a solution of the heat equation with initial condition $u_{0}$. For $u_{0} \in L^{p}, p>3$, we can use a similar but simpler method to find a lower bound on existence time that depends only on the norm of $u_{0}$ in $L^{p}$. This leads to estimates of the rate of putative blow-up of $\|u\|_{L^{p}}$ for $p>3$. Such estimates were known to Leray and a proof (based on the theory of semigroups) was given by Giga in [12] (see also [10]). Here we derive these classical estimates in an elementary way. These calculations can also be found in the paper by Beirão da Veiga [1], but we include them here for completeness since they are now also valid (due to our Lemma 2) in a periodic domain.

THEOREM 8. There exists an absolute constant $c>0$ such that if $\nabla \cdot u_{0}=0, u_{0} \in L^{p}, p>3$,

$$
T \leq c\left\|u_{0}\right\|_{L^{p}}^{-\frac{2 p}{p-3}}
$$

and $u$ is the solution of the Navier-Stokes equations with initial condition $u_{0}$, then $u$ is regular on the time interval $[0, T)$.

Proof. We multiply the Navier-Stokes equations by $u|u|^{\alpha-2}$ and integrate:

$$
\int u_{t} \cdot u|u|^{\alpha-2}-\int \Delta u \cdot u|u|^{\alpha-2}+\int(u \cdot \nabla) u \cdot u|u|^{\alpha-2}=-\int \nabla p \cdot u|u|^{\alpha-2} .
$$

The first term yields the time derivative of the $L^{\alpha}$ norm, since

$$
\int u_{t} \cdot u|u|^{\alpha-2}=\frac{1}{\alpha} \frac{\mathrm{d}}{\mathrm{d} t} \int|u|^{\alpha}
$$

Lemma 1 guarantees that

$$
-\int \Delta u \cdot u|u|^{\alpha-2} \geq \int|u|^{\alpha-2}|\nabla u|^{2}
$$


and the nonlinear term vanishes after an integration by parts,

$$
\begin{aligned}
\int(u \cdot \nabla) u \cdot u|u|^{\alpha-2} & =\frac{1}{2} \int|u|^{\alpha-2} \nabla|u|^{2} \cdot u \\
& =\frac{1}{\alpha} \int \nabla|u|^{\alpha} \cdot u \\
& =-\frac{1}{\alpha} \int|u|^{\alpha} \operatorname{div} u=0 .
\end{aligned}
$$

For the right-hand side

$$
\begin{aligned}
-\int \nabla p \cdot u|u|^{\alpha-2} & =\int p u \cdot \nabla|u|^{\alpha-2} \\
& =\frac{\alpha-2}{2} \int p|u|^{\alpha-4} u \cdot \nabla|u|^{2} \\
& \leq c \int|p||u|^{\alpha-2}|\nabla u| \\
& \leq c \int|p|^{2}|u|^{\alpha-2}+\frac{1}{2} \int|u|^{\alpha-2}|\nabla u|^{2}
\end{aligned}
$$

To estimate the pressure term we use Lemma 3,

$$
\begin{aligned}
c \int|p||u|^{\alpha-2} & \leq c\|p\|_{L^{\alpha}}^{2}\|u\|_{L^{\alpha}}^{\alpha-2} \\
& \leq c_{1}\|u\|_{L^{2 \alpha}}^{4}\|u\|_{L^{\alpha}}^{\alpha-2} \\
& \leq c_{1}\|u\|_{L^{\alpha}}^{\alpha-1}\|u\|_{L^{3 \alpha}}^{3} \\
& \leq \tilde{c}\|u\|_{L^{\alpha}}^{\alpha(\alpha-1) /(\alpha-3)}+\frac{1}{2 c_{\alpha}}\|u\|_{L^{3 \alpha}}^{\alpha} \\
& \leq \tilde{c}\|u\|_{L^{\alpha}}^{\alpha(\alpha-1) /(\alpha-3)}+\frac{1}{2} \int|u|^{\alpha-2}|\nabla u|^{2},
\end{aligned}
$$

using Lemma 2. Note that we can only use Young's inequality here when $\alpha>3$.

Combining the above estimates we get

$$
\frac{1}{\alpha} \frac{\mathrm{d}}{\mathrm{d} t}\|u\|_{L^{\alpha}}^{\alpha} \leq c\|u\|_{L^{\alpha}}^{\alpha(\alpha-1) /(\alpha-3)} .
$$

Thus $X=\|u\|_{L^{\alpha}}^{\alpha}$ satisfies the differential inequality

$$
\dot{X} \leq c X^{(\alpha-1) /(\alpha-3)} .
$$


A local smoothness criterion for solutions of the 3D Navier-Stokes etc. 177

With $\gamma=\frac{\alpha-1}{\alpha-3}$ it follows that

$$
Y^{\gamma-1}(t) \leq \frac{Y^{\gamma-1}(0)}{1-Y^{\gamma-1}(0)(\gamma-1) c^{\prime} t}
$$

as long as the denominator stays positive.

Corollary 9. There exists an absolute constant $c>0$ such that if $t=0$ is a blow up time for a solution $u$ of the Navier-Stokes equations (1) then

$$
\|u(-t)\|_{L^{p}} \geq c t^{-\frac{p-3}{2 p}}
$$

Note that the Sobolev embedding $\dot{H}^{s} \subset L^{6 /(3-2 s)}$ immediately implies a lower bound on blowup solutions in the homogeneous Sobolev space $\dot{H}^{s}$ for $1 / 2<s<3 / 2$.

Corollary 10. For $1 / 2<s<3 / 2$ there exists an absolute constant $c_{s}>0$ such that if $t=0$ is a blow up time for a solution $u$ of the NavierStokes equations (1) then

$$
\|u(-t)\|_{\dot{H}^{s}} \geq c t^{-(2 s-1) / 4} .
$$

We consider lower bounds in $\dot{H}^{s}$ for other values of $s$ in [18].

Acknowledgments. JCR and WS were supported by an EPSRC Leadership Fellowship EP/G007470/1. WS was also partially supported by the grant of the Polish Ministry of Science and Higher Education N N201 547438.

\section{REFERENCES}

[1] H. Beirão dA VeIGa. Existence and asymptotic behavior for strong solutions of the Navier-Stokes equations in the whole space, Indiana Univ. Math. J., 36 (1987), pp. 149-166.

[2] L.C. Berselli and G.P. Galdi. On the spacetime regularity of $C\left(0, T ; L^{n}\right)$ very weak solutions to the Navier-Stokes equations, Nonlinear Analysis, 58 (2004), pp. 703-717.

[3] M. CANNONE. Harmonic analysis tools for solving the incompressible NavierStokes equations, in S. Friedlander \& D. Serre (Eds.) Handbook of Mathematical Fluid Dynamics, vol. 3, Elsevier, 2003.

[4] J.-Y. Chemin, B. Desjardins, I. Gallagher and E. Grenier. Mathematical Geophysics, Oxford University Press, Oxford, 2006. 
[5] P. Constantin and C. Foias. Navier-Stokes equations. University of Chicago Press, Chicago, 1988.

[6] L. Escauriaza, G. Seregin, and V. Šverák. $L_{3, \infty}$-solutions of Navier-Stokes equations and backward uniqueness, Russian Math. Surveys, 58 (2003), pp. 211-250.

[7] L.C. Evans. Partial differential equations. Second edition. Graduate Studies in Mathematics, 19. American Mathematical Society, Providence, RI, 2010.

[8] R. FARWig, H. Sohr and W. VARnhorn. Necessary and sufficient conditions on local strong solvability of the Navier-Stokes system Applicable Analysis, Vol. 90, No. 1, January (2011), pp. 47-58.

[9] H. Fujita and T. Kato. On the Navier-Stokes initial value problem. I. Arch. Rational Mech. Anal., 16 (1964), pp. 269-315.

[10] G.P. GALDI. An introduction to the Navier-Stokes initial-boundary value problem, Fundamental directions in Mathematical Fluid Dynamics, Birkhauser, Basel, 1-70, 2011.

[11] G.P. GALDI and S. RIONERO. The weight function approach to uniqueness of viscous flows in unbounded domains, Arch. Ratl Mech. Anal., 69, 37 (1979).

[12] Y. GIGA. Solutions for semilinear parabolic equations in $L^{p}$ and regularity of weak solutions of the Navier-Stokes system. J. Differential Equations, 62 (1986), pp. 186-212.

[13] P.G. LEMARIÉ-RIEUsset. Recent developments in the Navier-Stokes problem, Chapman \& Hall/CRC Research Notes in Mathematics, 431. Chapman \& Hall/ CRC, Boca Raton, FL, 2002.

[14] T. Kato, Strong $L^{p}$-solutions of the Navier-Stokes equations in $\mathbb{R}^{m}$ with applications to weak solutions, Math. Zeit., 187 (1984), pp. 471-480.

[15] J. LERAY. Sur le mouvement d'un liquide visqueux emplissant l'espace, Acta Math., 63 (1934), pp. 193-248.

[16] P. Marín-Rubio, J.C. Robinson and W. SAdowski. Solutions of the $3 D$ Navier-Stokes equations for initial data in $H^{1 / 2}$ : robustness of regularity and numerical verification of regularity for bounded sets of initial data in $H^{1}$, J. Math. Anal. Appl., 400 (2013), pp. 76-85.

[17] A. Rodríguez Bernal. Introduction to semigroup theory for partial differential equations. Lectures notes, 2005. Available online at http:// www.opencontent.org/openpub/

[18] J.C. RoBInson, W. SADOWSKI and R. Silva. Lower bounds on blow up solutions of the three-dimensional Navier-Stokes equations in homogeneous Sobolev spaces, J. Math. Phys., 53 (2012), 115618.

[19] V. SHAPIRo. Fourier Series in Several Variables with Applications to Partial Differential Equations, Taylor \& Francis Group, LLC, Chapman \& Hall/CRC Applied Mathematics and Nonlinear Science Series Edt. Goong Chen, 2011.

[20] W. VON WAHL. Regularity of weak solutions of the Navier-Stokes equations. Nonlinear functional analysis and its applications, Part 2 (Berkeley, Calif., 1983), 497-503, Proc. Sympos. Pure Math., 45, Part 2, Amer. Math. Soc., Providence, RI, 1986.

Manoscritto pervenuto in redazione il 7 Ottobre 2012. 